\title{
Genetic polymorphisms may explain association between alcohol consumption and bladder cancer risk in East Asian men
}

\author{
Christian Daniel Fankhauser ${ }^{1}$, Hugh Mostafid $^{2}$ \\ ${ }^{1}$ Department of Urology, University Hospital, University of Zurich, Zurich, Switzerland; ${ }^{2}$ Department of Urology, Royal Surrey County Hospital, \\ Egerton Road, Guildford, Surrey, UK \\ Correspondence to: Hugh Mostafid. Department of Urology, Royal Surrey County Hospital, Egerton Road, Guildford, Surrey GU2 7XX, UK. \\ Email: Hugh.Mostafid@nhs.net. \\ Provenance: This is a Guest Editorial commissioned by Section Editor Xiao Li (Department of Urology, Jiangsu Cancer Hospital \& Jiangsu Institute \\ of Cancer Research \& Nanjing Medical University Affiliated Cancer Hospital, Nanjing, China). \\ Comment on: Masaoka H, Matsuo K, Sawada N, et al. Alcohol consumption and bladder cancer risk with or without the flushing response: The Japan \\ Public Health Center-based Prospective Study. Int J Cancer 2017;141:2480-8.
}

Submitted Apr 04, 2018. Accepted for publication Apr 11, 2018.

doi: $10.21037 /$ tau.2018.04.17

View this article at: http://dx.doi.org/10.21037/tau.2018.04.17

Bladder cancer (BC) is the second most common malignancy of the urinary tract, with an estimated 430,000 new diagnoses and 165,000 BC deaths per year worldwide (1). Unfortunately, only a few preventable risk factors for BC exist. Tobacco smoking represents the main risk factor for $\mathrm{BC}$ and $\mathrm{BC}$ incidence and mortality patterns and trends reflect corresponding smoking histories in each country (1). For example, the high BC incidence rates observed among US and Spanish men are likely to be the consequence of a very high smoking prevalence in the 1970s-1980s. Although Alcohol is a known risk factor for several malignancies, studies report conflicting results regarding $\mathrm{BC}$ incidence and alcohol consumption (2). Despite conflicting clinical data, the association between alcohol and $\mathrm{BC}$ has a biological rationale: alcohol is metabolized into several metabolites including acetaldehyde (ACE) which is carcinogenic.

In the article that accompanies this editorial Masaoka et al. (3) conducted a population-based cohort study using the Japan Public Health Center-based Prospective Study (JPHC study) (4) to investigate the association between facial flushing and BC risk stratified by alcohol consumption. Although previous epidemiological studies have examined the association between alcohol and $\mathrm{BC}$ risk in the USA and Europe and demonstrated no significant association (5) only two such studies have been conducted in Asia, both in Japan (5) where men have the highest incidence of BC in East Asia, and one of these showed a statistically significant increased risk of BC among alcohol drinkers (6). These findings indicate an association between alcohol consumption and BC risk in East Asian populations, contrary to Western populations, and might also account for the higher incidence of other alcohol-related cancers such as oesophageal cancer in these countries (7). Alcohol is metabolized to ACE which causes facial flushing and can also cause DNA damage and is carcinogenic (8). ACE is broken down by aldehyde dehydrogenase 2 (ALDH2). Genes encoding ALDH2 display polymorphism with varying and often reduced enzymatic activity and are known to differ in East Asian populations compared to the West. For example, approximately half of Japanese have the inactive ALDH2 Lys allele which shows significantly reduced enzymatic activity $(9,10)$ and is associated with the flushing response $(11,12)$. The authors therefore hypothesized that information on the flushing response can be used as a surrogate marker of ACE accumulation as a result of its reduced metabolism and reflect its carcinogenic effect on the bladder. Since ACE is excreted through the urinary tract (13) exposure of the urothelium to ACE may provide the explanation for an increased risk of $\mathrm{BC}$ associated with ACE accumulation in urine (14).

In this study the proportion of flushers in men decreased with increasing alcohol consumption and overall was not associated with increased BC risk. However, subgroup 
analysis showed that moderate drinkers with a flushing response had an increased risk of BC compared with non/ occasional and heavy drinkers. In contrast, no statistically significant association between alcohol drinking and BC risk was observed in men without the flushing response. To remove confounding by cigarette smoking the authors performed subgroup analysis on never smokers and showed that they had a similar association between alcohol consumption, flushing response and BC risk.

On the basis that ACE exposure plays an important role in the development of $\mathrm{BC}$ and facial flushing, if those with a flushing response all had the inactive ALDH2 Lys alleles one may expect that their risk of $\mathrm{BC}$ would increase with increasing alcohol consumption. However, the results show that among flushers the highest risk of $\mathrm{BC}$ is in moderate drinkers and not heavy drinkers. The authors suggest that these surprising findings may be explained by a poor correlation between self-reported facial flushing (which is very subjective) and ALDH2 genotype and indeed report several studies have shown that about $50 \%$ of flushers carry the $A L D H 2$ Glu/Glu gene $(15,16)$ whilst $86 \%$ of moderate drinkers with the flushing response carry the ALDH2 Lys gene and would be consistent with the suggestion that most moderate drinkers with a flushing response have ALDH2 Lys alleles, but that heavy drinkers with a flushing response include a considerable number of ALDH2 Glu/Glu carriers. This explanation appears to be supported by the author's own previous case-control study which showed that those with the ALDH2 Lys alleles who account for a large proportion of moderate drinkers had a higher risk of BC with increasing alcohol consumption, whereas those with ALDH2 Glu/Glu had no increase in risk even if they drank heavily (11) and this may attenuate the BC risk associated with heavy drinking.

Whilst these findings are interesting and point to genetic polymorphism as the underlying basis for epidemiological variations in different populations the study has several limitations. All subjects in the Tokyo area and some subjects in Osaka were excluded and since these are major centres of population in Japan, this may have had a bearing on the findings and their interpretation. In addition, the JPHC study relied on self-administered questionnaires with questions on lifestyle, alcohol consumption, flushing response after drinking alcohol and smoking habit and therefore under-reporting or over-reporting of the flushing response (which is very subjective and may be subject to recall bias) may have occurred. Finally, alcohol consumption and facial flushing was assessed differently between the two cohorts and the authors converted reported alcohol consumption to grams of pure ethanol per week both of which may inadvertently led to inaccuracies in the analysis. The authors recommend a prospective study to assess the role of $A L D H 2$ gene polymorphisms in ACE metabolism in an East Asian population to verify their hypothesis.

\section{Acknowledgements}

None.

\section{Footnote}

Conflicts of Interest: The authors have no conflicts of interest to declare.

\section{References}

1. Ferlay JS, Bray F, Forman D, et al. GLOBOCAN 2012, Cancer incidence and mortality worldwide. Lyon, France: International Agency for Research on Cancer, 2012.

2. Fankhauser CD, Mostafid H. Prevention of bladder cancer incidence and recurrence: nutrition and lifestyle. Curr Opin Urol 2018;28:88-92.

3. Masaoka H, Matsuo K, Sawada N, et al. Alcohol consumption and bladder cancer risk with or without the flushing response: The Japan Public Health Center-based Prospective Study. Int J Cancer 2017;141:2480-8.

4. Tsugane S, Sawada N. The JPHC study: design and some findings on the typical Japanese diet. Jpn J Clin Oncol 2014;44:777-82.

5. Pelucchi C, Galeone C, Tramacere I, et al. Alcohol drinking and bladder cancer risk: a meta-analysis. Ann Oncol 2012;23:1586-93.

6. Zaitsu M, Nakamura F, Toyokawa S, et al: Risk of Alcohol Consumption in Bladder Cancer: Case-Control Study from a Nationwide Inpatient Database in Japan. Tohoku J Exp Med 2016;239:9-15.

7. Ohashi S, Miyamoto S, Kikuchi O, et al. Recent Advances From Basic and Clinical Studies of Esophageal Squamous Cell Carcinoma. Gastroenterology 2015;149:1700-15.

8. IARC Working Group on the Evaluation of Carcinogenic Risks to Humans. Personal habits and indoor combustions. Volume $100 \mathrm{E}$. A review of human carcinogens. IARC Monogr Eval Carcinog Risks Hum 2012;100:1-538.

9. Crabb DW, Edenberg HJ, Bosron WF, et al. Genotypes for aldehyde dehydrogenase deficiency and alcohol sensitivity. The inactive ALDH2(2) allele is dominant. J 
Clin Invest 1989;83:314-6.

10. Gross ER, Zambelli VO, Small BA, et al. A personalized medicine approach for Asian Americans with the aldehyde dehydrogenase $2 * 2$ variant. Annu Rev Pharmacol Toxicol 2015;55:107-27.

11. Masaoka H, Ito H, Soga N, et al. Aldehyde dehydrogenase 2 (ALDH2) and alcohol dehydrogenase 1B (ADH1B) polymorphisms exacerbate bladder cancer risk associated with alcohol drinking: gene-environment interaction. Carcinogenesis 2016;37:583-8.

12. Asakage T, Yokoyama A, Haneda T, et al. Genetic polymorphisms of alcohol and aldehyde dehydrogenases, and drinking, smoking and diet in Japanese men with oral and pharyngeal squamous cell carcinoma. Carcinogenesis 2007;28:865-74.

13. Otsuka M, Harada N, Itabashi T, et al. Blood and urinary levels of ethanol, acetaldehyde, and C4 compounds such

Cite this article as: Fankhauser CD, Mostafid H. Genetic polymorphisms may explain association between alcohol consumption and bladder cancer risk in East Asian men. Transl Androl Urol 2018;7(Suppl 2):S252-S254. doi: 10.21037/ tau.2018.04.17 as diacetyl, acetoin, and 2,3-butanediol in normal male students after ethanol ingestion. Alcohol 1999;17:119-24.

14. Matsuo K, Wakai K, Hirose K, et al. Alcohol dehydrogenase 2 His47Arg polymorphism influences drinking habit independently of aldehyde dehydrogenase 2 Glu487Lys polymorphism: analysis of 2,299 Japanese subjects. Cancer Epidemiol Biomarkers Prev 2006;15:1009-13.

15. Ishiguro S, Sasazuki S, Inoue M, et al: Effect of alcohol consumption, cigarette smoking and flushing response on esophageal cancer risk: a population-based cohort study (JPHC study). Cancer Lett 2009;275:240-6.

16. Liu Y, Yoshimura K, Hanaoka T, et al. Association of habitual smoking and drinking with single nucleotide polymorphism (SNP) in 40 candidate genes: data from random population-based Japanese samples. J Hum Genet 2005;50:62-8. 